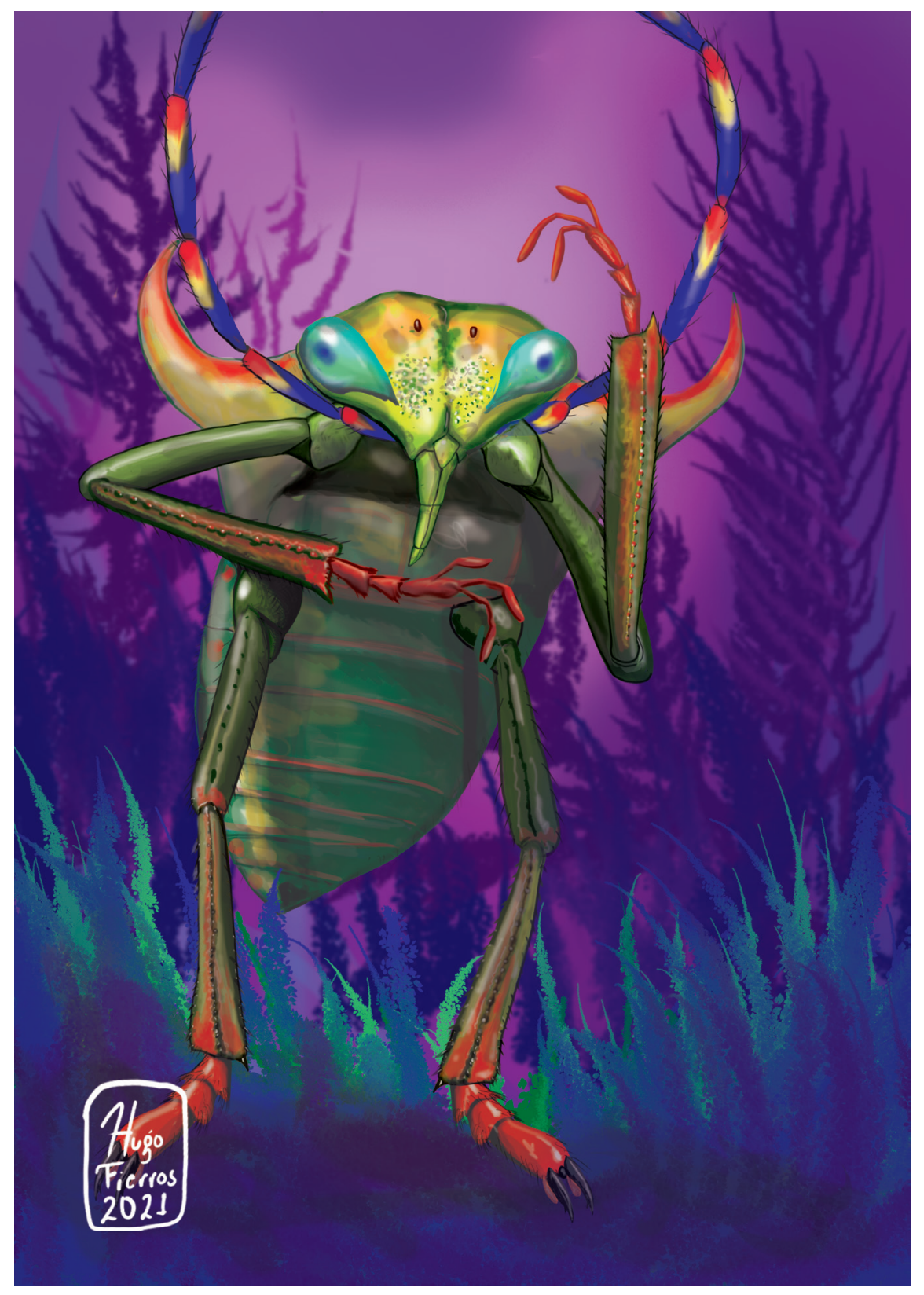

Dugesiana, Año 29, No. 1, (enero-junio, primer semestre 2022), es una publicación semestral, editada por la Universidad de Guadalajara, a través del Centro de Estudios en Zoología, por el Centro Universitario de Ciencias Biológicas y Agropecuarias. Camino Ramón Padilla Sánchez \# 2100, Nextipac, Zapopan, Jalisco, Tel. 37771150 ext. 33218, http://148.202.248.171/dugesiana/index.php/DUG/index, glenusmx@gmail.com. Editor responsable: José Luis Navarrete-Heredia. Reserva de Derechos al Uso Exclusivo 04-2009-062310115100-203, ISSN: 20079133, otorgados por el Instituto Nacional del Derecho de Autor. Responsable de la última actualización de este número: José Luis Navarrete-Heredia, Editor y Ana Laura González-Hernández, Asistente Editorial. Fecha de la última modificación 1 de enero de 2022, con un tiraje de un ejemplar.

Las opiniones expresadas por los autores no necesariamente reflejan la postura del editor de la publicación.

Queda estrictamente prohibida la reproducción total o parcial de los contenidos e imágenes de la publicación sin previa autorización de la Universidad de Guadalajara. 


\title{
Artículo
}

\section{Ensamble de coleópteros (Coleoptera) atraídos a la carroña en un bosque artificial resultado de la urbanización en el Área Natural Protegida Sierra de Guadalupe en el centro de México}

\author{
Ensembles of beetles (Coleoptera) attracted to carrion in an artificial forest result of the urbanization in \\ the Protected Natural Area Sierra de Guadalupe in central Mexico \\ Itzel Rodríguez-Castillo1, Esteban Jiménez-Sánchez ${ }^{* 1}$ y Jorge R. Padilla-Ramírez ${ }^{1}$
}

${ }^{1}$ Laboratorio de Zoología, Facultad de Estudios Superiores Iztacala, Universidad Nacional Autónoma de México. Av. de los Barrios 1, Los Reyes Iztacala, 54090, Tlalnepantla, Estado de México, México. oc.humdali@gmail.com, estjimsan@gmail.com, jorgepr@unam.mx

*Autor de correspondencia: estjimsan@gmail.com

oc.humdali@gmail.com, ORCID: https://orcid.org/0000-0001-6653-5305

estjimsan@gmail.com, ORCID: https://orcid.org/0000-0001-7310-7747

jorgepr@unam.mx, ORCID: https://orcid.org/0000-0002-6368-8397

\begin{abstract}
RESUMEN
Se estudio el ensamble de coleópteros, así como, la distribución estacional de la diversidad atraída a la carroña en un bosque artificial resultado de la urbanización del Área Natural Protegida Sierra de Guadalupe en el centro de México. Se realizaron muestreos mensuales de junio de 2017 a mayo de 2018 con el empleo de cuatro trampas tipo NTP-80 cebadas con carroña. Se capturaron un total de 3,434 individuos agrupados en 17 familias y 58 especies, de las cuales 13 se determinaron a nivel de especie y 45 a morfoespecie. Los estimadores de especies sugieren que se registró entre el $77.3 \%$ (Chao 1) y 80.5\% (ACE) de las especies. Las familias Staphylinidae, Silphidae, Carabidae, Leiodidae, Histeridae y Nitidulidae agruparon el $95.8 \%$ de la abundancia total y el $67.2 \%$ de las especies. La familia Staphylinidae presentó la mayor riqueza con 21 especies, las restantes tuvieron menos de cinco, de las cuales destacó la familia Silphidae con cuatro especies, la cual como gremio necrófago ocupo el segundo lugar en abundancia (21\%) después de los depredadores (62\%). La diversidad fue mayor en las lluvias que en la sequía, la similitud faunística entre épocas fue baja (J=0.37), con 32 especies exclusivas de las lluvias y cuatro de la sequía. En el área cohabitan especies de amplia distribución adaptadas a condiciones de perturbación, una especie endémica y convergen elementos de montaña con especies de ambientes semiáridos, por lo tanto, es importante mantener la conexión del bosque artificial con la zona de bosque de las partes altas de la Sierra de Guadalupe y conservar los fragmentos de matorral xerófilo que son muy escasos en la cuenca del Valle de México, para mitigar el impacto de la urbanización en la diversidad de coleópteros asociados a la carroña.
\end{abstract}

Palabras clave: NTP-80, Estado de México, Coacalco, Sierra de Guadalupe, necrófilos.

\begin{abstract}
The ensembles of beetles and the seasonal distribution of diversity attracted to carrion in an artificial forest result of the urbanization of the Protected Natural Area Sierra de Guadalupe in central Mexico was studied. Monthly samples were collected from June 2017 to May 2018 using four carrion traps type NTP-80. A total of 3,434 individuals belonging to 17 families and 58 species were collected, of which 13 were identified to the species level and 45 to morphospecies. The species estimators suggest that was registered between $77.3 \%$ (Chao 1) and $80.5 \%$ (ACE) of the species. The families Staphylinidae, Silphidae, Carabidae, Leiodidae, Histeridae and Nitidulidae grouped $95.8 \%$ of the total abundance and $67.2 \%$ of the species. The family Staphylinidae was the higher richness with 21 species, the remaining had less than five species, of which the family Silphidae was the most important with four species, and represented the necrophagous guilt which was the second most abundant making up $21 \%$ of all beetles collected after the predators $(62 \%)$. The diversity was higher in rainy than in drought season, the faunistic similarity between seasons was low $(\mathrm{J}=0.37)$, with 32 species exclusive of rainy and four of drought season. In the area inhabit wide-ranging species adapted to perturbation conditions, an endemic species, and converge montane elements with species of semiarid environments, therefore, is important to maintain the connection of the artificial forest with the forest area in upper parts of the Sierra de Guadalupe, and conserve the fragments of xeric shrubland that are scarce in the basin of Mexican Valley, so as to mitigate the impact of the urbanization in the diversity of beetles associated to carrion.
\end{abstract}

Key words: carrion trap, Estado de México, Coacalco, Sierra de Guadalupe, necrophilous. 
La urbanización ha provocado la desaparición de muchos de los hábitats de las especies especialistas las cuales han sido reemplazadas por especies generalistas adaptadas a ambientes artificiales creados por el hombre (parques urbanos y jardines), los cuales ofrecen refugio a especies nativas y nuevos colonizadores (Sánchez-Bayo y Wyckhuys, 2019). En estos sitios la biodiversidad puede verse incrementada por la adición de especies exóticas introducidas por el hombre de forma accidental o intencional, que pueden desplazar rápidamente a las especies nativas, además estos hábitats artificiales frecuentemente tienen una productividad primaria mucho mayor que los ecosistemas naturales adyacentes, debido al aporte constante de agua, fertilizantes y generación de residuos orgánicos. El incremento de la riqueza de especies es muy evidente en plantas, en contraste con las especies de invertebrados, donde parece no haber un incremento paralelo (McKinney 2008), debido a que, aun cuando las comunidades de plantas urbanas pueden ser muy diversas, en muchos casos, más diversas que los ecosistemas naturales, no han evolucionado con las especies de artrópodos nativas (Dale y Frank 2018).

En la Sierra de Guadalupe, Estado de México, el matorral xerófilo corresponde a la vegetación nativa de las zonas más bajas (2,240 msnm) de la Sierra, sin embargo, ha desaparecido debido a la urbanización siendo sustituido por la introducción de especies arbóreas exóticas en programas de reforestación desde hace más de 50 años, creando bosques artificiales con escasos elementos del matorral; en la parte más alta de la Sierra y las cañadas de difícil acceso, aún se conserva el bosque de encino nativo (GCDMX 2016). Estas alteraciones indudablemente afectan la fauna de coleópteros asociados a la carroña, los cuales como muchos insectos juegan un papel importante en la descomposición y reciclaje de la materia orgánica necesaria para proveer de nutrientes para el crecimiento de las plantas (Schowalter et al. 2018), sin embargo, se desconoce la diversidad que alberga esta área.

Por lo anterior, el objetivo es conocer la composición de coleópteros atraídos a la carroña en un bosque artificial producto de la reforestación, así como, la distribución temporal de la diversidad. Al tratarse de un área afectada por la urbanización, se espera que el número de familias y especies de coleópteros sea menor que el encontrado en áreas naturales no perturbadas. Por otro lado, el área se caracteriza por tener un periodo de lluvias y uno sequía bien definidos, lo cual determinará una distribución estacional marcada para las poblaciones de coleópteros.

\section{MATERIAL Y MÉTODOS}

Área de estudio. La Sierra de Guadalupe es una Área Natural Protegida localizada al norte de la Ciudad de México y los límites del Estado de México. El muestreo se realizó en la periferia de la Sierra, dentro del "Centro Ecoturístico y de Educación Ambiental Sierra de Guadalupe" ubicado en el municipio de Coacalco de Berriozábal, Estado de
México en los $19^{\circ} 36^{\prime} 37^{\prime \prime} \mathrm{N}$ y 99 $95^{\circ} 20^{\prime \prime O}$ (Figura 1A). Pertenece a la región hidrológica del Río Pánuco de la cuenca del Río Moctezuma y a la provincia fisiográfica Faja Volcánica Transmexicana, tiene un rango altitudinal que va de 2,240 a 2,800-3,000 msnm, y es un pequeño conjunto de elevaciones volcánicas independientes y sobrepuestas (Lugo-Hubp y Salinas-Montes 1996). Predomina un clima templado subhúmedo con lluvias en verano $\mathrm{Cb}(\mathrm{w} 0)(\mathrm{w})(\mathrm{i}$ ') g; presenta temperatura media anual de $16.7^{\circ} \mathrm{C}$, enero es el mes más frío con una media de $13.1^{\circ} \mathrm{C}$ y junio el más cálido con $18.8^{\circ} \mathrm{C}$ (Cedillo et al. 2007). Con precipitación media anual de $733.9 \mathrm{~mm}$, enero es el más seco con $30 \mathrm{~mm}$ y junio el más húmedo con $152 \mathrm{~mm}$ (GCDMX 2016). El periodo de sequía va de noviembre a abril y el de lluvias de mayo a octubre (Peña 2018).

Los muestreos se hicieron en una zona fuertemente afectada por la urbanización, donde se realizan actividades recreativas como: campismo, senderismo, deporte y pastoreo por parte de los habitantes de la zona urbana de los alrededores. En este lugar se presenta un bosque artificial con asociaciones vegetales derivadas de las perturbaciones y la introducción de especies exóticas, producto de la reforestación con fines de restauración y conservación (Cedillo et al. 2007; GCDMX 2016) (Figura 1B). El estrato forestal estuvo compuesto por plantaciones de Eucalyptus camaldulensis Dehnh. (eucalipto), Casuarina equisetifolia L. (casuarina), Cupressus lindleyi Klotzsch ex Endl. (cedro blanco), Cupressus sempervirens L. (ciprés panteonero) y diversas especies de pinos, entre las que se encuentran Pinus cembroides Zucc., P. montezumae Lamb., P. patula Schltdl. \& Cham. y P. radiata D.Don. El estrato de sotobosque está dominado por plantas herbáceas anuales de Poaceae, Asteraceae, Fabaceae y Convolvulaceae, con escasos elementos del matorral xerófilo nativo como Cactaceae: Opuntia sp. (nopales), Mammillaria sp. (biznaga), Asparagaceae: Agave sp. (maguey) y Fabaceae: Vachellia farnesiana (L.) Wight \& Arn. (huizache). Las zonas de mayor altitud y las cañadas de difícil acceso son las mejor conservadas y donde se puede encontrar bosque natural de encino (GCDMX 2016).

Diseño del muestreo. Para la captura se colocaron cuatro trampas tipo NTP-80 (Morón y Terrón 1984) modificadas, cebadas con calamar el cual fue sustituido mensualmente y como líquido conservador monoetilenglicol (Figura 1C). Las trampas se ubicaron a nivel de suelo y se distribuyeron en cuatro puntos en un transecto de aproximadamente un kilómetro a lo largo de la ladera norte del cerro (sitio 1: 2,455 msnm, $19^{\circ} 36^{\prime} 27^{\prime \prime}$ N, $99^{\circ} 05^{\prime} 35^{\prime \prime}$ O; sitio 2: 2,472 msnm, $19^{\circ} 36^{\prime} 24.6^{\prime \prime} \mathrm{N}, 99^{\circ} 05^{\prime} 33.5^{\prime \prime} \mathrm{O}$; sitio 3: 2,502 msnm, $19^{\circ} 36^{\prime} 25.9^{\prime \prime} \mathrm{N}, 99^{\circ} 05^{\prime} 30.2^{\prime \prime} \mathrm{O}$; sitio 4: 2,531 msnm, $19^{\circ} 36^{\prime} 20.2^{\prime \prime} \mathrm{N}, 99^{\circ} 05^{\prime} 28.7^{\prime}$ ' O) con una diferencia de $25 \mathrm{msnm}$ y $150 \mathrm{~m}$ de distancia en promedio entre cada trampa (Figura 1A). Las trampas estuvieron instaladas permanentemente de junio de 2017 a mayo de 2018, las muestras se recuperaron cada mes y se colocaron en frascos con etanol al 70\% para su traslado al laboratorio. 
Procesamiento del material entomológico. El material obtenido fue separado y determinado hasta el nivel taxonómico posible, mediante claves especializadas (Arnett y Thomas 2001; Arnett, Jr. et al. 2002; NavarreteHeredia et al. 2002; Triplehorn y Johnson 2005; Delgado 2008; Navarrete-Heredia 2009; Márquez y Asiain 2010; Moctezuma y Halffter 2020) y por comparación con especímenes depositados en la Colección de Artrópodos de la FES Iztacala (CAFESI), UNAM, donde se depositó todo el material. El $77.6 \%$ de las especies que correspondió a 45 morfoespecies, fueron determinados a nivel de género, subfamilia o familia, y solamente 13 a nivel de especie, a lo largo del escrito se hace referencia a todas ellas en conjunto con el termino de especies. Para organizar las familias se empleó la clasificación de Bouchard et al. (2011).

Análisis de datos. Se contó el número de individuos (abundancia) y número de especies (riqueza) por mes y por sitio. La eficiencia del muestreo se evaluó con los estimadores de especies no paramétricos ACE y Chao 1, para lo cual se utilizó el programa Estimates versión 9 (Colwell y Coddington 1994). Se elaboró la curva de rangoabundancia para evaluar la estructura cuantitativa de la comunidad. Además, se calculó el índice de diversidad de Shannon (H) y la uniformidad (E) por época del año, se aplicó la prueba de $t$ de Hutchenson para saber si había diferencias significativas de la diversidad entre épocas. La similitud entre épocas se evaluó con el índice de similitud de Jaccard (J), todos estos cálculos se realizaron con el programa Past versión 4.0.1. (Hammer et al. 2001). Los gremios tróficos para cada familia están basados en Thayer (2005), Stork y Grimbacher (2006), Davis et al. (2011) y Moctezuma y Halffter (2020). Las morfoespecies determinadas hasta nivel de subfamilia o género permitieron una asignación un poco más precisa a cada uno de los gremios tróficos, tal fue el caso de las familias Staphylinidae, Scarabaeidae y Curculionidae, las cuales son muy diversas e incluyen taxones con hábitos alimentarios particulares; en el caso de las morfoespecies determinadas a nivel de familia el gremio fue asignado con base en el gremio predominante en las especies de la familia, por lo que puede existir un margen de error en su asignación.

\section{RESULTADOS}

Se obtuvo un total de 3,434 individuos agrupados en 17 familias y 58 especies (Cuadro 1). Los estimadores de especies sugieren que se registró entre el 77.3\% (Chao 1= $75)$ y $80.5 \%(\mathrm{ACE}=72)$ de las especies necrófilas del área (Figura 2).

Las familias Staphylinidae, Silphidae, Carabidae, Leiodidae, Histeridae y Nitidulidae (ordenadas de mayor a menor abundancia) agruparon el 95.8\% (3,292 individuos) de todos los coleópteros capturados y el 67.2\% (39 especies) de las especies. La familia Staphylinidae fue la de mayor riqueza con 21 especies, seguida de Carabidae e Histeridae con cinco cada una; Silphidae y Tenebrionidae tuvieron cuatro especies cada una, las restantes familias tuvieron menos de tres (Cuadro 1).

Aleocharinae sp. 1 (970 individuos), Nicrophorus mexicanus Matthews, 1888 (651 individuos) y Carabidae sp. 2 (606 individuos) fueron las especies dominantes y en conjunto representaron el $64.8 \%$ de la abundancia total; Leiodidae sp. 1, Xerosaprinus sp. y Nitidulidae sp. 1 tuvieron entre 130 y 310 individuos cada una y agruparon el $19.6 \%$ de la abundancia; 15 especies registraron entre diez y noventa individuos, las restantes 37 tuvieron menos de diez (Figura 3).

El mayor promedio mensual de riqueza y abundancia se registró en junio y julio, y los valores más bajos fueron en diciembre y enero (Figura 4A-B). En la temporada de lluvias se obtuvo mayor riqueza (54 especies) y abundancia (2,903 individuos) que durante la sequía (26 especies y 531 individuos). De las 16 familias registradas en las lluvias, 12 también estuvieron presentes en la sequía. La familia Anthicidae fue la única exclusiva de la sequía; mientras que Chrysomelidae, Hydrophilidae, Ptiliidae y Scarabaeidae fueron exclusivas de la época de lluvias (Cuadro 1). La similitud entre la época de lluvias y la de sequía fue baja con $37 \%(\mathrm{~J}=0.37)$, con veintidós especies presentes en ambas épocas, 32 fueron exclusivas de las lluvias y cuatro de la sequía (Cuadro 1). La diversidad fue significativamente mayor en las lluvias $(\mathrm{H}=2.1)$ que en la sequía $(\mathrm{H}=2)(\mathrm{p}=$ 0.001) (Figura 5A). Por el contrario, la uniformidad fue mayor en la sequía $(E=0.28)$ que en las lluvias $(E=0.16)$ (Figura 5B).

A mitad del periodo de sequía (febrero- marzo) se observó un pico de abundancia (Figura 4A), el cual se debió principalmente a las especies de Histeridae: Xerosaprinus sp. y Saprinus sp. que mostraron preferencia por los meses secos (Cuadro 1).

De la familia Scarabaeidae, la especie Ontophagus chevrolati Harold, 1869 fue exclusiva de las lluvias. En el caso de la familia Silphidae, N. mexicanus se recolectó todo el año, mientras que Oxelytrum discicolle (Brullé, 1836) y Thanatophilus truncatus (Say, 1823) (Silphidae), fueron casi exclusivos del periodo húmedo, solo capturados esporádicamente en los meses secos. La especie Thanatophilus graniger (Chevrolat, 1833) no mostró un patrón definido debido a su baja abundancia en ambos periodos. Finalmente, de las 21 especie de Staphylinidae, solamente Aleocharinae sp. 1 se registró todo el año, las especie restantes fueron exclusivas de las lluvias (Cuadro 1).

En cuanto a los gremios tróficos, los depredadores representaron el $62 \%$ de la abundancia total, seguidos por los necrófagos (21\%) y los fungívoros (14\%) (Figura 6A). En cuanto a la riqueza, los gremios mejor representados también fueron los depredadores que agruparon a la mitad de las especies (50\%), le siguieron los fungívoros (19\%) y los saprófagos generalistas (12\%); los necrófagos, aunque ocuparon el segundo lugar de abundancia, solo agruparon al 8\% de las especies (Figura 6B). Tanto la abundancia como el número de especies de los diferentes gremios 
tróficos disminuyó durante la sequía, con excepción de la abundancia de los herbívoros y la riqueza de los necrófagos que fueron similares en ambas épocas; mientras que la abundancia de los saprófagos generalistas fue ligeramente mayor en la sequía (Fig. 6C y D).

\section{DISCUSIÓN}

Son pocos los estudios llevados a cabo en México que proporcionan datos sobre la totalidad de familias de coleópteros recolectadas con trampas cebadas con carroña, entre éstos se encuentra el de Morón y Terrón (1984) quienes al igual que en el presente estudio donde se registraron 17 familias, también obtuvieron un número bajo de familias con 20, en un área cubierta por bosques mesófilo y tropical perturbados en la Sierra Norte de Hidalgo.

Lo anterior contrasta con lo observado en otras localidades de México en lugares con problemáticas similares a la Sierra de Guadalupe, relacionadas con la substitución de la vegetación original por especies exóticas, como el bosque urbano de Los Colomos, Jalisco, ubicado a $1,551 \mathrm{~m}$, con clima templado semicálido donde obtuvieron 28 familias (Navarrete-Heredia et al. 2012); o la zona de Teotihuacan cercana a la Sierra de Guadalupe, con altitud similar $(2,300 \mathrm{~m})$ pero con un clima semiseco templado, con áreas cubiertas por extensos cultivos, zonas urbanas y suburbanas, con escasos fragmentos de matorral xerófilo nativo, donde se registraron 33 familias (Moreno-Olvera et al. 2014). Por otro lado, en una zona conservada NaranjoLópez y Navarrete-Heredia (2011) registraron 34 familias en dos localidades, una en un bosque tropical caducifolio y otra en un bosque de pino en Gómez Farías, Jalisco, a una altitud de $1,678 \mathrm{~m}$ con clima semiseco y semicálido respectivamente. En todos los casos mencionados, se trata de muestreos anuales realizados con un número variable de trampas tipo NTP-80, que va desde cuatro como en el presente estudio hasta 14, abarcando dos o más áreas con características de vegetación, clima y altitud diferente. Labrador (2005) encontró que el número máximo de familias registradas en la carroña en México es de 38.

De acuerdo con los estimadores, es posible que un mayor esfuerzo de recolecta permita la captura de entre 19\% y $22 \%$ de especies adicionales en la Sierra de Guadalupe, lo cual podría incrementar también el número de familias. Aun así, la composición de familias observada en la Sierra de Guadalupe, es consistente con aquellas que tienen mayor afinidad por la carroña como es el caso de: Staphylinidae, Silphidae, Carabidae, Leiodidae, Histeridae, Nitidulidae, Scarabaeidae, Trogidae y Tenebrionidae (Shubeck et al. 1977; Morón y Terrón 1984; Naranjo-López y NavarreteHeredia 2011; Navarrete-Heredia et al. 2012; MorenoOlvera et al. 2014), las cuales también se han recolectado en estudios de interés forense (Almeida y Mise 2009).

Las familias Anthicidae, Chrysomelidae, Cryptophagidae, Curculionidae, Hydrophilidae, Latridiidae, Mycetophagidae y Ptiliidae correspondieron al $47 \%$ del total de familias, todas ellas con excepción de
Anthicidae (28 individuos) y Curculionidae (18 individuos) tuvieron menos de diez individuos, lo cual coincide con lo observado en la mayoría de los estudios (Shubeck et al. 1977; Shubeck et al. 1981; Morón y Terrón 1984; Labrador, 2005; Naranjo-López y Navarrete-Heredia 2011; NavarreteHeredia et al. 2012; Moreno-Olvera 2015), donde entre el $33 \%$ y $57 \%$ de las familias están representadas por menos de diez individuos; las familias mencionadas han sido registradas comúnmente en trampas cebadas con carroña por lo que podrían ser consideradas como secundarias por su baja abundancia.

De los curculiónidos registrados, solo se capturó un individuo perteneciente a la subfamilia Scolytinae (Manarthrum sp.) cuyas especies se caracterizan por ser de hábito xilófago/fungívoro, de manera general los escolitinos podrían considerarse accidentales en la carroña, si bien Scolytinae han sido registrada en gran abundancia en la mayoría de los estudios llevados a cabo en el país (Morón y Terrón 1984; Naranjo-López y Navarrete-Heredia 2011; Navarrete-Heredia et al. 2012), esto se debe a un artefacto causado por los componentes de la mezcla utilizada como líquido conservador, el cual está formado principalmente por alcohol etílico como lo sugiere el diseño original de la trampa NTP-80 (Morón y Terrón, 1984), esto simula la fermentación de la madera y puede atraer grandes cantidades de escolitinos (Burgos-Solorio 1998; Navarrete-Heredia et al. 2012; Quezada-García et al. 2014), mientras que en el presente estudio la captura de un solo escolitino se debe a que el líquido conservador utilizado fue monoetilenglicol el cual es casi inodoro.

La mayor riqueza y abundancia de coleópteros registrada durante las lluvias es un patrón común de la fauna asociada a la carroña en el país (Rodríguez et al. 2018), lo cual coincide con la mayor disponibilidad de recursos; de manera general se observó que las especies más abundantes estuvieron presentes prácticamente todo el año como fue el caso de $N$. mexicanus, aunque su mayor abundancia se observó en el periodo de lluvias, salvo algunas excepciones como ocurrió con Xerosaprinus sp. y Saprinus sp. que prefirieron la sequía; en este sentido, se ha visto que la familia Histeridae destaca por su preferencia por la sequía independientemente del tipo de vegetación donde sea recolectada, ya sea bosque tropical caducifolio, bosque de pino, matorral xerófilo o bosques urbanos (Naranjo-López y Navarrete-Heredia 2011; Navarrete-Heredia et al. 2012; Moreno-Olvera et al. 2014), en algunos estudios se ha detectado que aunque los histéridos pueden estar representados por hasta cinco especies como ocurrió en el presente estudio, solamente las de los géneros Xerosaprinus y Saprinus muestran clara preferencia por la sequía (Naranjo-López y NavarreteHeredia 2011).

La familia Scarabaeidae fue exclusiva de las lluvias y solo se registraron dos especies, un individuo de Orizabus clunalis (LeConte, 1856) (Dynastinae), la cual tiene amplia distribución y habita en bosques mesófilo, pino, encino, matorral y comunidades secundarias establecidas entre 
los 500 y 3,000 m de altitud (Ratcliffe y Morón, 1997), fue considerada accidental debido a su hábito fitófago; también se recolectaron 29 individuos de Onthophagus chevrolati (Scarabaeinae) que es una especie copronecrofaga que habita en bosques de encino-pino y encino, aunque se ha visto que es más abundante en matorral, pastizal y vegetación secundaria en bosques desmontados en la cercanía de zonas boscosas, además, esta especie es un elemento de montaña característico de la Zona de Transición Mexicana distribuida entre los 1,500 y 4,000 m de altitud (Zunino y Halffter 1988; Halffter et al. 2019; Moctezuma y Halffter 2020). En ambos casos su presencia en la Sierra de Guadalupe coincide con las características de altitud y vegetación conocidas para estas especies.

A pesar de que el área de muestreo no fue muy extensa y la perturbación observada en la Sierra de Guadalupe, se registraron cuatro especies de tres géneros distintos de Silphidae, que corresponde al máximo número de especies registradas en otros estudios del país (Navarrete-Heredia y Quiroz-Rocha 2000; Quiroz-Rocha et al. 2008; TrevillaRebollar et al. 2010; Naranjo-López y Navarrete-Heredia 2011; García-Fernández y Jiménez-Sánchez 2014; Jiménez Sánchez et al. 2019; Reyes-Hernandez y Navarrete-Heredia 2019, Rodríguez et al. 2019).

Al comparar la composición de especies obtenidas en la Sierra de Guadalupe con el estudio llevado a cabo en la zona de Teotihuacán (Jiménez Sánchez et al. 2019), ubicada también en la cuenca del Valle de México, se encontró que compartió las mismas especies de Silphidae y Trogidae; para la primera familia destaca Thanatophilus graniger (Chevrolat, 1833) que es endémica de México la cual ha sido registrada en las provincias de la Sierra Madre oriental y el Cinturón Volcánico Transmexicano (Márquez et al. 2015) y en el caso de Trogidae solo se registró a Trox plicatus Robinson, 1840 que es una especie ampliamente distribuida desde el sur de Estados Unidos hasta el centrosur de México (Deloya 2000), se tienen registros puntuales de la especie en bosques de mesófilo de montaña, encino, encino-pino y matorral xerófilo entre los 2,000 y 2,400 msnm (Morón y Deloya 1991; Pérez-Villamares et al. 2016; Reyes-Hernandez y Navarrete-Heredia 2019), por lo que su presencia coincide con el rango altitudinal y los tipos de vegetación presentes en la región.

Sin considerar a las especies de la subfamilia Aleocharinae, la Sierra de Guadalupe (15 especies) tuvo casi el mismo número de especies de Staphylinidae que las capturadas con la trampa NTP-80 en la región de Teotihuacán (16 especies) (Jiménez Sánchez et al. 2019) y compartió especies que habitan principalmente ambientes semiáridos como fue el caso de: Belonuchus ephippiatus (Say, 1830) (Smetana 1995; Ferro et al. 2013; JiménezSánchez et al. 2013) y Belonuchus erichsoni Bernhauer, 1917, sin embargo, en la Sierra de Guadalupe también se encontraron especies representativas de las zonas templadas que podrían ser consideradas como elementos de montaña, tal es el caso de Philonthus testaceipennis Erichson, 1840 y Tachinus mexicanus Campbell, 1973 (Campbell 1973; Márquez y Asiain 2010; Rodríguez et al. 2019), cuyas poblaciones posiblemente sean más abundantes en el bosque de encino que se encuentra en las zonas más altas de la sierra.

Por lo tanto, el bosque artificial de la Sierra de Guadalupe aloja especies tanto de la zona semiárida que abarca la mayor parte de la cuenca del valle de México, así como, especies de montaña del área de bosque de encino presente en las partes altas de la Sierra cuyo pico máximo puede alcanzar los 3,000 m (Lugo-Hubp y Salinas-Montes 1996), además en esta área se tiene una alta riqueza de especies de Silphidae que son muy importantes en la degradación y reciclamiento de los cadáveres, así mismo, este grupo de necrófagos fue el segundo gremio más abundante, mientras que los depredadores ocuparon el primer lugar en cuanto a número de especies y abundancia. Trumbo y Bloch (2000) encontraron que los sílfidos del género Nicrophorus fueron menos exitosos en encontrar y enterrar los cadáveres en fragmentos de bosque pequeños que en áreas más grandes de bosque; por lo que, en el área de estudio la conexión del bosque artificial que aún existe con el área boscosa mayor que cubre las partes altas de la Sierra, probablemente determinan la alta riqueza de especies de Silphidae registrada y las poblaciones grandes de $N$. mexicanus.

Por último, aunque la diversidad de familias en la Sierra de Guadalupe es baja comparada con otros estudios, la presencia de especies tanto de ambientes semiáridos como de zonas templadas denotan la importancia que tiene el conservar la vegetación nativa aledaña como son los bosques de encino de la parte alta de la sierra y los fragmentos de matorral xerófilo que cada vez son más escasos en la cuenca del Valle de México, las cuales mantienen las poblaciones de coleópteros presentes en las áreas con impacto de la urbanización. Además, es urgente ordenar el uso del suelo, debido a que los datos indican que en la Sierra de Guadalupe el suelo urbano ha tenido un incremento lineal y se ha cuadruplicado en los últimos treinta años (Peña 2018).

\section{AGRADECIMIENTOS}

A Gabriela Medina, Andrés Oropeza Petrich, Mónica Copihue Vega Ávila, Raquel Castillo Flores y Daniel G. Torres Millán, así como, a los guardaparques del Parque Estatal Sierra de Guadalupe por su apoyo durante los muestreos. A William David Rodríguez por sus valiosos comentarios que enriquecieron el manuscrito. Este estudio fue parcialmente apoyado por el Proyecto Zoología de la División de Investigación y Posgrado de la FES Iztacala, UNAM.

\section{LITERATURA CITADA}

Almeida, L.M and K.M Mise. 2009. Diagnosis and key of the main families and species of South American Coleoptera of forensic importance. Revista Brasileira de Entomologia, 53 (2): 227-244. 
Arnett, Jr. Ross H, and Michael C Thomas. 2001. Volume 1. American Beetles. Archostemata, Myxophaga, Adephaga, Polyphaga: Staphyliniformia. United States of America: CRC Press.

Arnett, Jr., Ross H., Michael C. Thomas, Paul E. Skelley, and J Howard Frank. 2002. Volume 2. American Beetles. Polyphaga: Scarabaeoidea through Curculionoidea. Edited by Arnett Jr Ross H., Michael C Thomas, Paul E Skelley, and J Howard Frank. America. United States of America: CRC Press.

Bouchard, P., Y. Bousquet, A.E. Davies, M.A. AlonsoZarazaga, J.F. Lawrence, C.H.C. Lyal, A.F. Newton, C.A.M. Reid, M. Schmitt, S.A. Slipiński and A.B.T. Smith. 2011. Family-group names in Coleoptera (Insecta). ZooKeys, 972 (88): 1-972. https://doi. org/10.3897/zookeys.88.807.

Burgos-Solorio, A. 1998. Escarabajos barrenadores Platypodidae y Scolytidae (Coleoptera) atraídos a trampas NTP-80 de La Cañada de los alrededores de San José de Los Laureles Talayacapan, Morelos, México. Dugesiana, 5(2): 29-34.

Campbell, J.M. 1973. A Revision of the genus Tachinus (Coleoptera: Staphylinidae) of North and Central America. Memoirs of the Entomological Society of Canada, 105(S90): 7-137. https://doi.org/10.4039/ entm10590fv.

Cedillo, O.L., M.A. Rivas y F.N. Rodríguez. 2007. El Área Natural Protegida Sujeta a Conservación Ecológica "Sierra de Guadalupe". Revista Sistemas Ambientales, 1(1): 1-14.

Colwell, R.K., and J.A. Coddington. 1994. Estimating terrestrial biodiversity through extrapolation. Philosophical Transactions of the Royal Society of London. Series B, Biological Sciences, 345(1311): 10118. https://doi.org/10.1098/rstb.1994.0091.

Dale, A.G. and S.D. Frank. 2018. Urban plants and climate drive unique arthropod interactions with unpredictable consequences. Current Opinion in Insect Science, 29: 27-33. https://doi.org/10.1016/j.cois.2018.06.001.

Davis, A.J., S.L. Sutton and M.J.D. Brendell. 2011. Vertical distribution of beetles in a tropical rainforest in Sulawesi : The role of the canopy in contributing to biodiversity. Sepilok Bulletin, 13\&14: 59-83.

Delgado, L. 2008. A New Species of Orizabus (Coleoptera: Scarabaeidae) from Mexico, new records, and a revised key to the species. Annals of the Entomological Society of America, 101(1): 53-57. https://doi.org/10.1603/00138746(2008)101[53:ANSOOC]2.0.CO;2.

Deloya, C. 2000. Distribución de la familia Trogidae en México (Coleoptera: Lamellicornia). Acta Zoologica Mexicana (n.s.), 81: 63-76.

Ferro, M.L., N. Nguyen, A. Tishechkin, J.-S. Park, V. Bayless, and C.E. Carlton. 2013. Coleoptera collected from rotting fishhook barrel cacti (Ferocactus wislizeni (Engelm.) Britton and Rose), with a review of nearctic Coleoptera associated with succulent necrosis. The
Coleopterists Bulletin, 67(4): 419-43. https://doi. org/10.1649/0010-065x-67.4.419.

García-Fernández, C. y E. Jiménez-Sánchez. 2014. Coleópteros necrófilos (Coleoptera: Scarabaeidae, Silphidae y Trogidae) de La Sierra de Santa Rosa y El Marfil, Guanajuato, México. Entomología Mexicana, 1: 464-469.

Gobierno de la Ciudad de México - GCDMX. 2016. Programa de manejo del Área Natural Protegida, con categoría de Zona Sujeta a Conservación Ecológica "Sierra de Guadalupe". Gaceta Oficial de la Ciudad de México.

Halffter, G., M. Zunino, V. Moctezuma and J.L. SánchezHuerta. 2019. The integration processes of the distributional patterns in the mexican transition zone: phyletic, paleogeographic and ecological factors of a case study. Zootaxa, 4586(1): 1-34. https://doi. org/10.11646/zootaxa.4586.1.1.

Hammer, Ø., D.A.T. Harper and P.D. Ryan. 2001. Past: paleontological statistics software package for education and data analysis. Palaeontologia Electronica, 4(4): 5-7. https://doi.org/10.1016/j.bcp.2008.05.025.

Jennings, V.H. and D.W. Tallamy. 2006. Composition and abundance of ground-dwelling Coleoptera in a fragmented and continuous forest. Environmental Entomology, 35(6): 1550-1560. https://doi. org/10.1603/0046-225X(2006)35[1550:CAAOGC]2.0. $\mathrm{CO} ; 2$.

Jiménez-Sánchez, E., R. Quezada-García y J. PadillaRamírez. 2013. Diversidad de escarabajos necrófilos (Coleoptera: Scarabaeidae, Silphidae, Staphylinidae y Trogidae) en una región semiárida del valle de Zapotitlán de Las Salinas, Puebla, México. Revista de Biología Tropical, 61(3): 1475-1491.

Jiménez-Sánchez, E., R. Quezada-García, J.R. PadillaRamírez, M.L. Moreno and M.A. Angel. 2019. Variación de la diversidad de Staphylinidae, Silphidae y Trogidae (Insecta: Coleoptera) en un gradiente urbano-agrícola en una región semiárida del Estado de México, México. Acta Zoológica Mexicana (n.s.), 35: 1-16. https://doi. org/10.21829/azm.2019.3502152.

Labrador, G. 2005. Coleópteros necrófilos de México distribución y diversidad. Tesis de licenciatura. Universidad de Guadalajara, Jalisco, México.

Lugo-Hubp, José y A. Salinas-Montes. 1996. Geomorfología de la Sierra de Guadalupe (al norte de la Ciudad de México) y su relación con peligros naturales. Revista Mexicana de Ciencias Geológicas, 13(2): 240-251.

Márquez, J. and J. Asiain. 2010. Three new species of the Philonthus furvus species group (Coleoptera: Staphylinidae) from Guatemala and México, with taxonomic remarks and distributional records of related mexican species. Transactions of the American Entomological Society, 136(3+4): 269-288.

Márquez, J., J. Escoto-Rocha, and I. Goyenechea. 2015. Distribution patterns of the mexican species of carrion 
beetles (Coleoptera: Silphidae). The Coleopterists Bulletin, 69(4): 813-823. https://doi.org/10.1649/0010065X-69.4.813.

McKinney, M.L. 2008. Effects of urbanization on species richness: a review of plants and animals. Urban Ecosystems, 11(2):161-176. https://doi.org/10.1007/ s11252-007-0045-4.

Moctezuma, V. and G. Halffter. 2020. New species and redescriptions of the Onthophagus chevrolati species complex (Coleoptera: Scarabaeoidea: Scarabaeinae). Annales Zoologici, 70(2): 245-261. https://doi.org/10.3 161/00034541ANZ2020.70.2.005.

Moreno-Olvera, M.L., E. Jiménez-Sánchez y J. PadillaRamírez. 2014. Coleópteros (Insecta: Coleoptera) necrófilos de una región semiárida en el nororiente del Estado de México, México. Entomología Mexicana, 1:1073-1078.

Moreno, M.L. 2015. Coleópteros (Coleoptera: Insecta) necrófilos de sitios con diferentes usos de suelo de una región semiárida en el nororiente del Estado de México. Tesis de licenciatura. Facultad de Estudios Superiores Iztacla, UNAM. Estado de México, México.

Morón, M.A. y C. Deloya. 1991. Los coleópteros lamelicornios de la Reserva de la Biosfera 'La Michilia, Durango, México. Folia Entomológica Mexicana, 81: 209-283.

Morón, M.A. and R.A. Terrón. 1984. Distribución altitudinal y estacional de los insectos necrófilos en la Sierra Norte de Hidalgo, México. Acta Zoologica Mexicana (n.s.), 3: 1-47.

Naranjo-López, A.G., and J.L. Navarrete-Heredia. 2011. Coleópteros necrócolos (Histeridae, Silphidae y Scarabaeidae ) en dos localidades de Gómez Farías, Jalisco, México. Revista Colombiana de Entomología, 37(1): 103-110.

Navarrete-Heredia, J.L. 2009. Silphidae (Coleóptera) de México: diversidad y distribución. Universidad de Guadalajara, Jalisco, México.

Navarrete-Heredia, J.L. y G.A. Quiroz-Rocha. 2000. Macro-coleopteros necrófilos de San Jose de Los Laureles, Morelos, México (Coleoptera: Scarabaeidae y Silphidae). Folia Entomológica Mexicana, 110: 1-13.

Navarrete-Heredia, J.L., A.F. Newton, M.K. Thayer, J.S. Ashe and D.S. Chandler. 2002. Guía ilustrada para los géneros de Staphylinidae (Coleoptera) de México. Universidad de Guadalajara y CONABIO, México.

Navarrete-Heredia, J.L., C.I. Sainz, A.L. GonzálezHernández, G.A. Quiroz-Rocha, A. Hernández, M. Vásquez-Bolaños, D. Vega-Romero and B. Hernández. 2012. Coleópteros necrócolos del bosque Los Colomos, Guadalajara, Jalisco, México. Dugesiana, 19(2): 157162.

Peña, A.K. 2018. Análisis del cambio espacio-temporal de la cobertura vegetal dentro de la Sierra de Guadalupe, México, mediante teledetección. Tesis de maestría. Centro interdisciplinario de Investigaciones y Estudios sobre Medio Ambiente y Desarrollo (CIIEMAD), Instituto Politécnico Nacional, México.

Pérez-Villamares, J.C., E. Jiménez-Sánchez y J.R. Padilla-Ramírez. 2016. Escarabajos atraídos a la carroña (Coleoptera: Scarabaeidae, Geotrupidae, Hybosoridae, Trogidae y Silphidae) en las cañadas de Coatepec Harinas, Estado de México, México. Revista Mexicana de Biodiversidad, 87(2): 443-50. https://doi. org/10.1016/j.rmb.2016.03.005.

Quezada-García, R., E. Jiménez-Sánchez, A. EquihuaMartínez y J. Padilla-Ramírez. 2014. Escolitinos y platipodinos ( Coleoptera: Curculionidae ) atraídos a trampas tipo NTP-80 en Zapotitlán de Las Salinas, Puebla, México. Acta Zoologica Mexicana (n.s.), 30(3): 625-636.

Quiroz-Rocha, G.A., J.L. Navarrete-Heredia y P.A. Martínez. 2008. Especies de Scarabaeinae (Coleoptera: Scarabaeidae) y Silphidae (Coleoptera) necrófilas de bosque de pino-encino y bosque mesófilo de montaña en el municipio Mascota, Jalisco, México. Dugesiana, 15(1): 27-37.

Ratcliffe, B.C y M.A. Morón. 1997. Capítulo 3. Dynastinae. (pp. 53-163). En: Morón, M.A., B.C. Ratcliffe y C. Deloya (Eds.). Atlas de los escarabajos de México. CONABIO-Sociedad Mexicana de Entomología A.C. Veracruz, México.

Reyes-Hernández, J.L. and J.L. Navarrete-Heredia. 2019. Species assemblage of carrion beetles (Coleoptera: Scarabaeidae Silphidae, Trogidae) in three habitats in the northeastern region of Aguascalientes, Mexico. The Coleopterist Bulletin, 73(3): 646-654.

Rodríguez, W.D., J.L. Navarrete-Heredia and J. Klimaszewski. 2018. Rove beetles collected with carrion traps (Coleoptera: Staphylinidae) in Quercus forest of Cerro de García, Jalisco and Quercus, Quercuspine, and pine forests in other jurisdictions of Mexico. Zootaxa, 4433(3): 457-77. https://doi.org/10.11646/ zootaxa.4433.3.4.

Rodríguez, W.D., J.L. Navarrete-Heredia, R. RodríguezMacías, G. Briceño-Félix, M. Vásquez-Bolaños and J. Klimaszewski. 2019. Diversity patterns of necrocolous beetles (Coleoptera: Scarabaeidae, Silphidae, Trogidae) in Agave tequilana Weber (Asparagaceae) fields of different ages. The Canadian Entomologist, 151(5): 585-599. doi:10.4039/tce.2019.29

Sánchez-Bayo, F. and K.A.G. Wyckhuys. 2019. Worldwide decline of the entomofauna: a review of its drivers. Biological Conservation, 232: 8-27. https://doi. org/10.1016/j.biocon.2019.01.020.

Schowalter, T.D., J.A. Noriega y T. Tscharntke. 2018. Insect effects on ecosystem services - introduction. Basic and Applied Ecology, 26: 1-7. https://doi.org/10.1016/j. baae.2017.09.011.

Shubeck, P.P., N.M. Downie and R.L. Wenzel. 1977. Species composition of carrion beetles in a mixed-oak forest. Memorial Forest Bulletin, 4: 12-17. 
Shubeck, P.P., N.M. Downie, R.L. Wenzel and S.B. Peck. 1981. Species composition and seasonal abundance of carrion beetles in an oak-beech forest in the Great Swamp National Wildlife Refuge (N.J.). Entomological News, 92(1): 7-16.

Smetana, A. 1995. Rove beetles of the subtribe Philonthina of America North of Mexico (Coleoptera: Staphylinidae): classification, phylogeny, and taxonomic revision. American Entomological Institute, USA.

Stork, N.E. and P.S. Grimbacher. 2006. Beetle assemblages from an australian tropical rainforest show that the canopy and the ground strata contribute equally to biodiversity. Proceedings of the Royal Society B: Biological Sciences, 273(1596):1969-1975. https://doi. org/10.1098/rspb.2006.3521.

Recibido: 21 de septiembre 2021

Aceptado: 26 de noviembre 2021
Thayer, M.K. 2005. Staphylinidae Latreille, 1802. (Pp. 296-344). In: Beutel R.G. and R.A.B. Leschen (Eds.). Coleoptera, Beetles. Volume 1: Morphology and Systematics (Archostemata, Adephaga, Myxophaga, Polyphaga Partim). Walter de Gruyter, Berlin, Germany.

Trevilla-Rebollar, A., C. Deloya and J.R. Padilla-Ramírez. 2010. Coleópteros necrófilos (Scarabaeidae, Silphidae y Trogidae) de Malinalco, Estado de México, México. Neotropical Entomology, 39(4): 486-495.

Triplehorn, Charles A., and Norman F. Johnson. 2005. Borror and Delong's Introduction to the Study of Insects. Seventh Ed. USA: Thomson Brooks/Cole.

Trumbo, S.T. and P.L. Bloch. 2000. Habitat fragmentation and burying beetle abundance and success. Journal of Insect Conservation, 4: 245-252.

Zunino, M. and G. Halffter. 1988. Análisis taxonómico, ecológico y biogeográfico de un grupo americano de Onthophagus (Coleoptera: Scarabaeidae). Museo Regionale Di Scienze Naturali, Monografie, (9): 1-211.
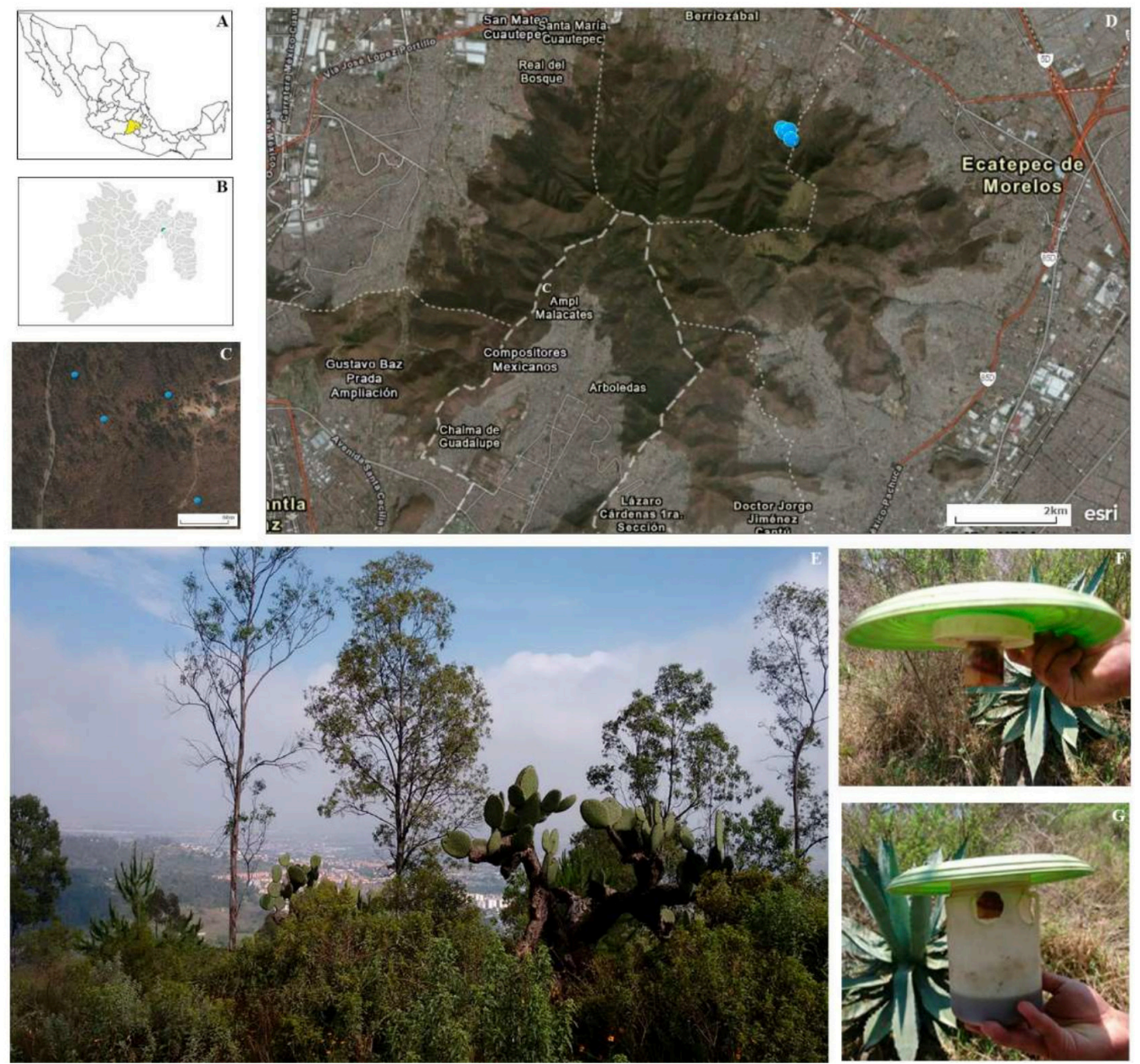

Figura 1. Área de estudio y diseño de la trampa. Ubicación del área de estudio: A. Nivel nacional, B. Nivel estatal, C. Sitios de muestreo, D. Perspectiva de la Sierra de Guadalupe con puntos de muestreo (puntos azules), E. Panorámica de la vegetación de la zona de muestreo. Trampa NTP-80 modificada: F. Tapa y frasco con el cebo (calamar) y G. Trampa ensamblada. Fotos por E. Jiménez e I. Rodríguez. 
Figura 2. Curvas de acumulación de especies de coleópteros observadas (línea negra), y estimadas Chao1 (línea gris) y ACE (línea discontinua).

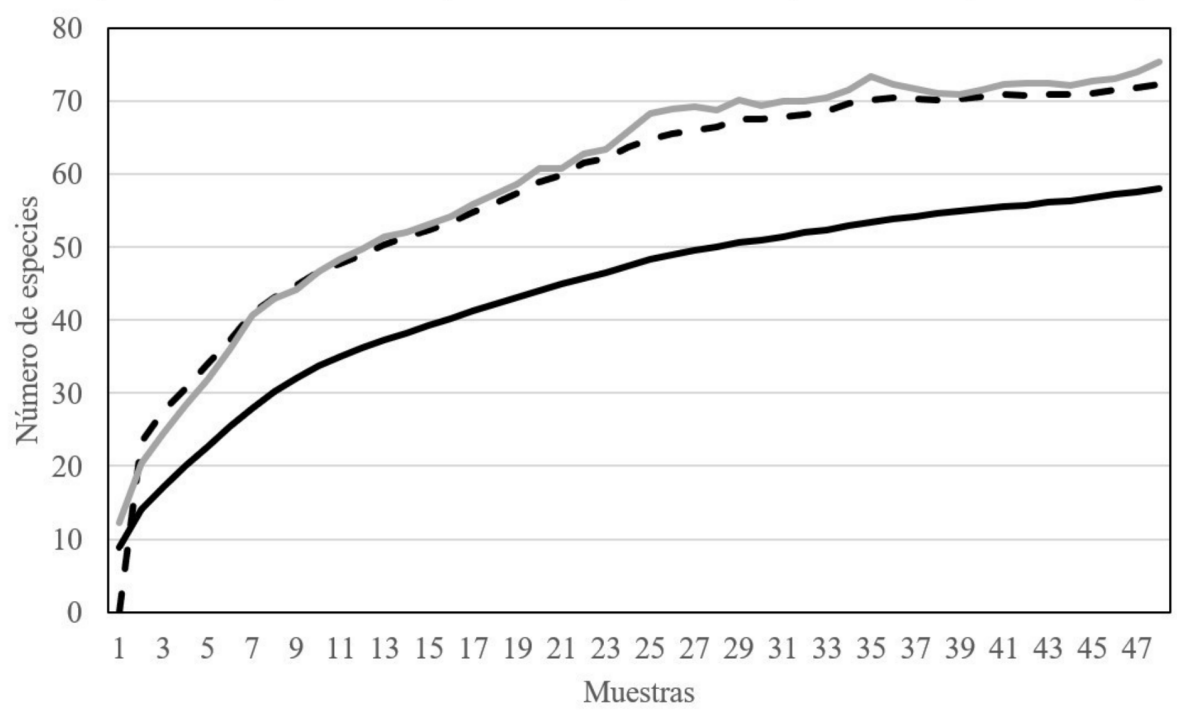

Figura 3. Curva de rango abundancia del ensamble de coleópteros asociados a la carroña.

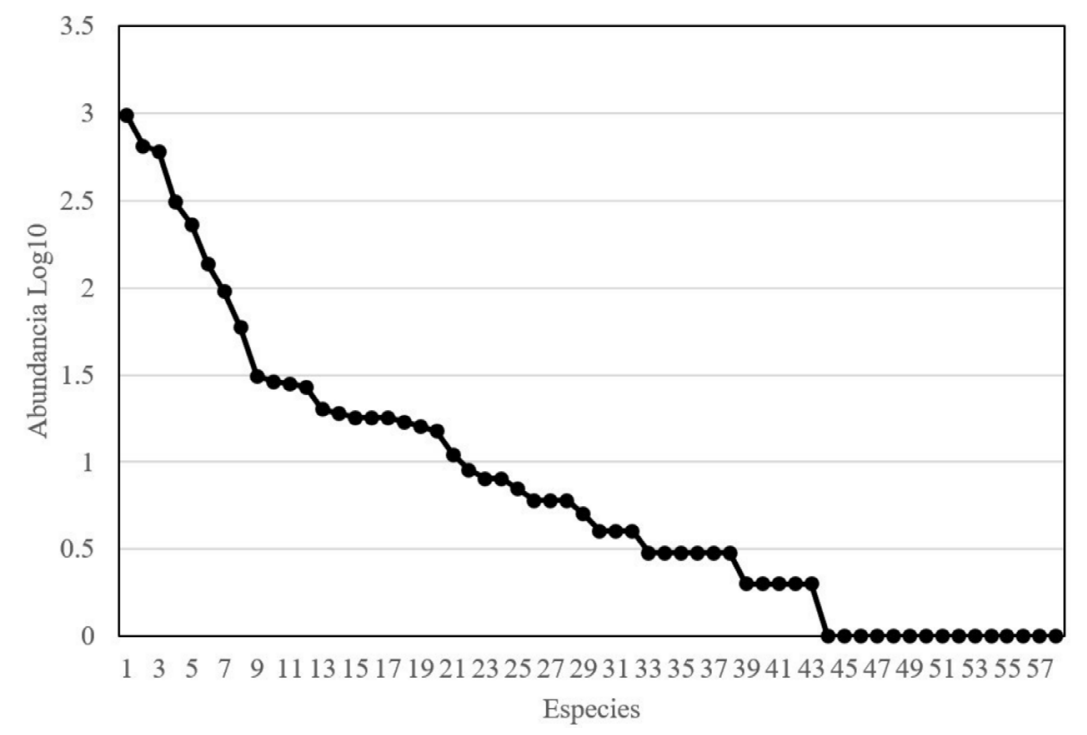

A

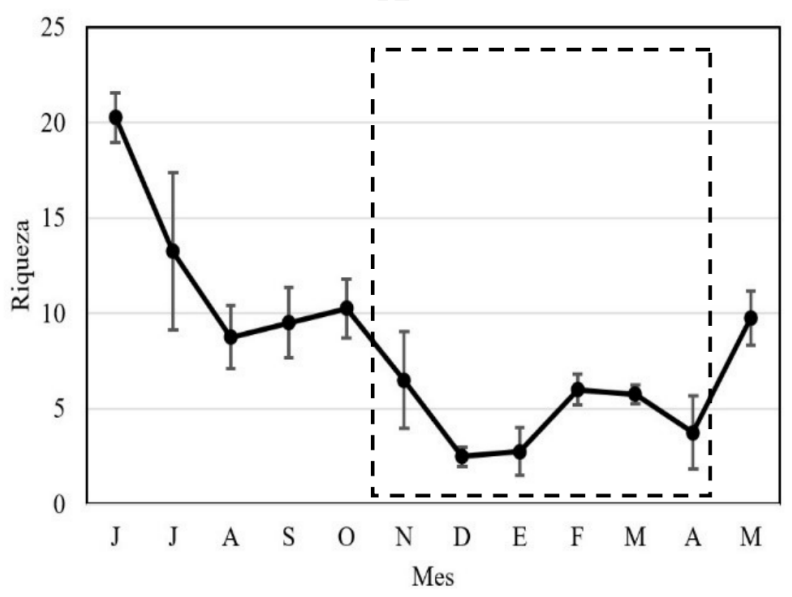

B

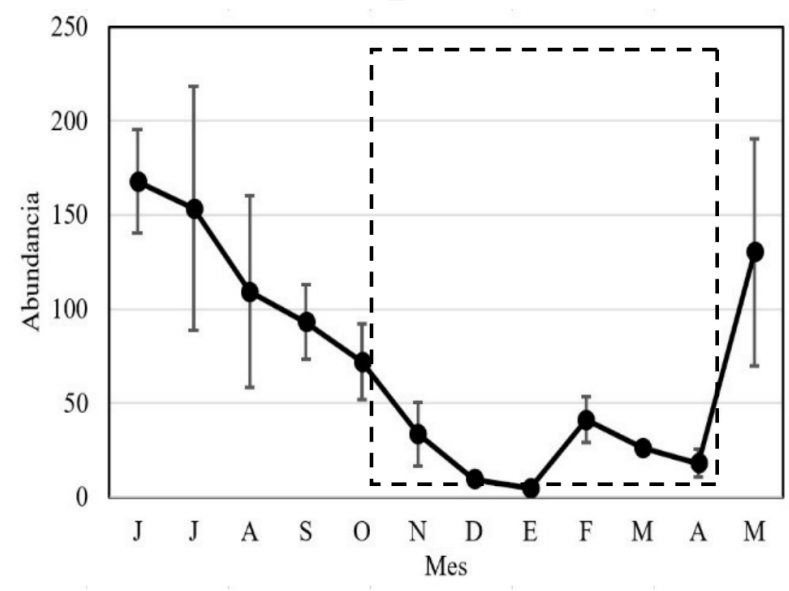

Figura 4. Promedio mensual \pm error estándar del número de especies (A) e individuos (B) de coleópteros asociados a la carroña. Los meses dentro del rectángulo punteado corresponden a la época de sequía. 
A

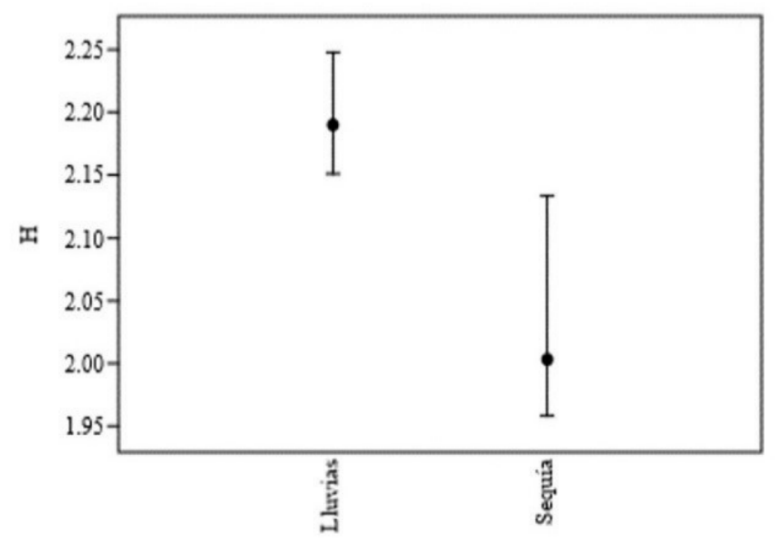

B

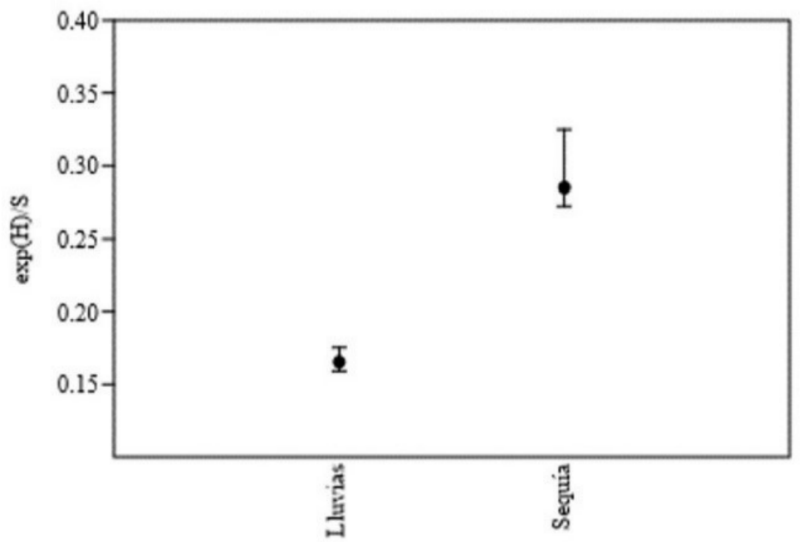

Figura 5. Diversidad (A) y uniformidad (B) de coleópteros asociados a la carroña en la época de lluvias y sequía. Las barras de error corresponden al intervalo de confianza de $95 \%$.

A

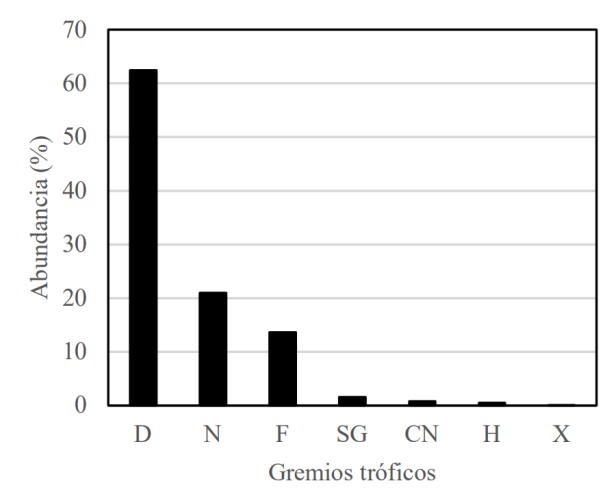

C

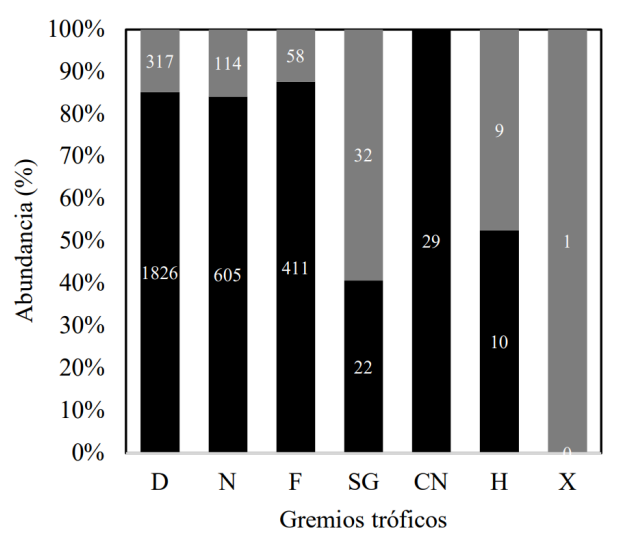

B

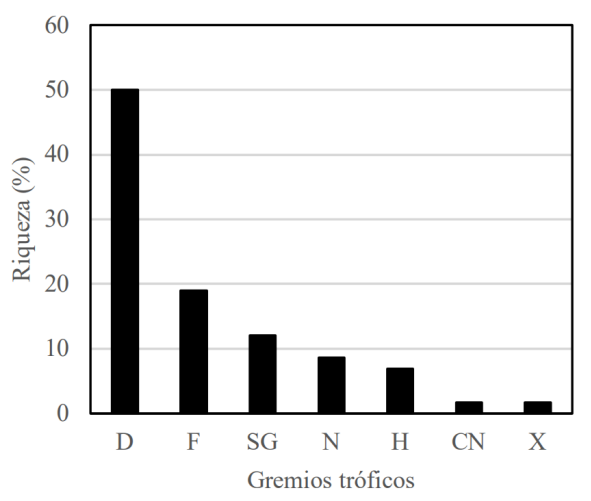

D

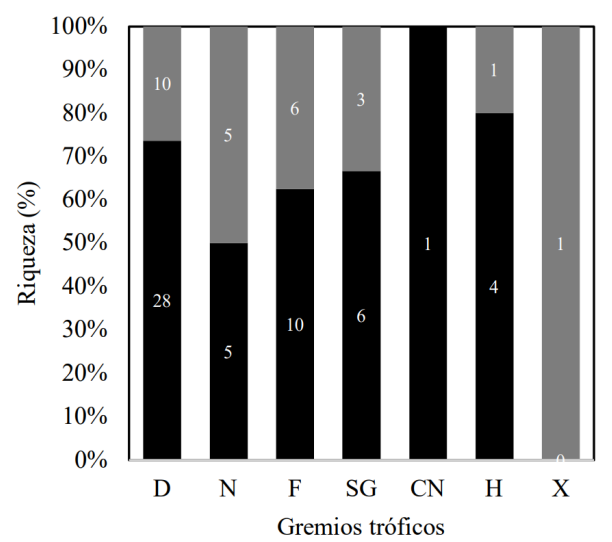

Figura 6. Porcentaje de abundancia $(A, C)$ y riqueza $(B, D)$ de los gremios tróficos de coleópteros asociados a la carroña durante el ciclo anual (A, B), en la estación de lluvias (barra negra) y en la sequía (barra gris) (C, D). D = depredador; $\mathrm{F}$ = fungívoro; $\mathrm{SG}=$ saprófago-generalista; $\mathrm{N}=$ necrófago; $\mathrm{H}=$ herbívoro; $\mathrm{CN}=$ copro-necrófago; $\mathrm{X}=$ xilófago. 
Cuadro 1. Abundancia y riqueza mensual de los coleópteros asociados a carroña en la Sierra de Guadalupe. Las columnas sombreadas corresponden a la época de sequía. $\mathrm{LL}=$ lluvias; $\mathrm{S}=$ sequía; $\mathrm{GT}$ = gremio trófico. Meses J-M = julio-mayo. En la columna de gremios tróficos: $\mathrm{D}=$ depredador; $\mathrm{F}$ = fungívoro; $\mathrm{SG}=$ saprófago generalista; $\mathrm{N}=$ necrófago; $\mathrm{H}=$ herbívoro; $\mathrm{C}-\mathrm{N}=$ copro-necrófago; $\mathrm{X}=$ xilófago. * Familia exclusiva de la sequía; **Familia exclusiva de las lluvias.

\begin{tabular}{|c|c|c|c|c|c|c|c|c|c|c|c|c|c|c|c|c|}
\hline Taxones & $\mathbf{J}$ & $\mathbf{J}$ & $\mathbf{A}$ & $\mathbf{S}$ & $\mathbf{O}$ & $\mathbf{N}$ & D & $\mathbf{E}$ & $\mathbf{F}$ & M & $\mathbf{A}$ & M & $\mathbf{L L}$ & S & Total & GT \\
\hline Anthicidae & & & & & & 9 & 8 & 5 & 6 & & & & & $28 *$ & 28 & \\
\hline Anthicidae sp. 1 & & & & & & 9 & 8 & 5 & 6 & & & & & 28 & 28 & $\mathrm{H}, \mathrm{SG}$ \\
\hline Carabidae & 181 & 184 & 92 & 19 & 11 & 3 & & 1 & & 1 & 17 & 169 & 656 & 22 & 678 & \\
\hline Carabidae sp. 1 & 1 & & & & & & & & & & & & 1 & & 1 & $\mathrm{D}$ \\
\hline Carabidae sp. 2 & 163 & 145 & 86 & 14 & 11 & 2 & & 1 & & 1 & 16 & 167 & 586 & 20 & 606 & $\mathrm{D}$ \\
\hline Carabidae sp. 3 & 11 & 35 & 6 & 5 & & & & & & & 1 & 1 & 58 & 1 & 59 & $\mathrm{D}$ \\
\hline Elaphrini sp. & 6 & 4 & & & & & & & & & & 1 & 11 & & 11 & $\mathrm{D}$ \\
\hline Promecognatinni_sp. & & & & & & 1 & & & & & & & & 1 & 1 & $\mathrm{D}$ \\
\hline Chrysomelidae & 1 & & & & & & & & & & & & $1 * *$ & & 1 & \\
\hline Alticini sp. 1 & 1 & & & & & & & & & & & & 1 & & 1 & $\mathrm{H}$ \\
\hline Cryptophagidae & 2 & & & & & 1 & 1 & & & 3 & & 1 & 3 & 5 & 8 & \\
\hline Cryptophagidae sp. 1 & 1 & & & & & & & & & 3 & & & 1 & 3 & 4 & $\mathrm{~F}$ \\
\hline Cryptophagidae sp. 2 & 1 & & & & & & & & & & & 1 & 2 & & 2 & $\mathrm{~F}$ \\
\hline Cryptophagidae sp. 3 & & & & & & 1 & 1 & & & & & & & 2 & 2 & $\mathrm{~F}$ \\
\hline Curculionidae & 2 & 1 & & & 2 & 3 & & 2 & 4 & 1 & & 3 & 8 & 10 & 18 & \\
\hline Monarthrum sp. & & & & & & & & & 1 & & & & & 1 & 1 & $\mathrm{X} / \mathrm{F}$ \\
\hline Curculionidae sp. 1 & 1 & 1 & & & 2 & 3 & & 2 & 3 & 1 & & 3 & 7 & 9 & 16 & $\mathrm{H}$ \\
\hline Curculionidae sp. 2 & 1 & & & & & & & & & & & & 1 & & 1 & $\mathrm{H}$ \\
\hline Histeridae & 29 & 6 & 4 & 3 & 44 & 63 & 12 & 6 & 32 & 47 & 18 & 39 & 125 & 178 & 303 & \\
\hline Eremosaprinus_sp. & 10 & 2 & 1 & & & & & & & & 1 & 1 & 14 & 1 & 15 & $\mathrm{D}$ \\
\hline Euspilotus sp. & 5 & 2 & 2 & & & & & & & & & & 9 & & 9 & $\mathrm{D}$ \\
\hline Geomysaprinus sp. & 2 & 2 & 1 & 2 & 9 & 1 & & & & & & 1 & 17 & 1 & 18 & $\mathrm{D}$ \\
\hline Saprinus sp. & 6 & & & & 3 & 2 & & 1 & 7 & 3 & & 9 & 18 & 13 & 31 & $\mathrm{D}$ \\
\hline Xerosaprinus sp. & 6 & & & 1 & 32 & 60 & 12 & 5 & 25 & 44 & 17 & 28 & 67 & 163 & 230 & $\mathrm{D}$ \\
\hline Hydrophilidae & & 1 & & & & & & & & & & & $1 * *$ & & 1 & \\
\hline Hydrophilidae sp. & & 1 & & & & & & & & & & & 1 & & 1 & SG \\
\hline Latridiidae & 1 & & & & & & & & & 3 & & 2 & 3 & 3 & 6 & \\
\hline Latridiidae sp. 1 & 1 & & & & & & & & & 3 & & 2 & 3 & 3 & 6 & $\mathrm{~F}$ \\
\hline Leiodidae & 135 & 57 & 67 & 34 & 15 & & & & 1 & & & 2 & 310 & 1 & 311 & \\
\hline Leiodidae sp. 1 & 135 & 57 & 67 & 34 & 14 & & & & 1 & & & 2 & 309 & 1 & 310 & $\mathrm{~F}$ \\
\hline Leiodidae sp. 2 & & & & & 1 & & & & & & & & 1 & & 1 & $\mathrm{~F}$ \\
\hline Mycetophagidae & & & & & & 2 & & & & & & 3 & 3 & 2 & 5 & \\
\hline Mycetophagidae sp. 1 & & & & & & 2 & & & & & & 3 & 3 & 2 & 5 & $\mathrm{~F}$ \\
\hline Nitidulidae & 27 & 13 & 2 & 1 & 14 & 5 & 3 & 1 & 27 & 8 & 3 & 33 & 90 & 47 & 137 & \\
\hline Nitidulidae_sp. 1 & 26 & 13 & 2 & 1 & 14 & 5 & 3 & 1 & 27 & 8 & 3 & 33 & 89 & 47 & 136 & $\mathrm{~F}$ \\
\hline Nitidulidae sp. 2 & 1 & & & & & & & & & & & & 1 & & 1 & $\mathrm{~F}$ \\
\hline Ptiliidae & 1 & & & & & & & & & & & & 1 & & 1 & \\
\hline Ptiliidae sp. 1 & 1 & & & & & & & & & & & & $1 * *$ & & 1 & $\mathrm{~F}$ \\
\hline Scarabaeidae & 24 & 5 & & 1 & & & & & & & & & $30 * *$ & & 30 & \\
\hline Onthophagus chevrolati & 24 & 4 & & 1 & & & & & & & & & 29 & & 29 & $\mathrm{C}-\mathrm{N}$ \\
\hline Orizabus clunalis & & 1 & & & & & & & & & & & 1 & & 1 & $\mathrm{H}$ \\
\hline Silphidae & 148 & 21 & 100 & 194 & 93 & 45 & 10 & 1 & 15 & 17 & 25 & 30 & 586 & 113 & 699 & \\
\hline Nicrophorus mexicanus & 136 & 20 & 94 & 192 & 79 & 40 & 9 & 1 & 15 & 16 & 24 & 25 & 546 & 105 & 651 & $\mathrm{~N}$ \\
\hline Oxelytrum discicolle & 3 & 1 & 5 & 1 & 12 & 2 & & & & & 1 & 2 & 24 & 3 & 27 & $\mathrm{~N}$ \\
\hline Tanatophilus graniger & & & & & & & 1 & & & 1 & & 1 & 1 & 2 & 3 & $\mathrm{~N}$ \\
\hline Tanatophilus truncatus & 9 & & 1 & 1 & 2 & 3 & & & & & & 2 & 15 & 3 & 18 & $\mathrm{~N}$ \\
\hline Staphylinidae & 113 & 319 & 167 & 110 & 105 & & 4 & 2 & 78 & 24 & 9 & 233 & 1047 & 117 & 1164 & \\
\hline Aleochara sp. 1 & & & & & 1 & & & & & & & & 1 & & 1 & $\mathrm{D}$ \\
\hline Aleochara sp. 2 & & & & & & & & & 2 & & & 1 & 1 & 2 & 3 & $\mathrm{D}$ \\
\hline Aleocharinae sp. 1 & 58 & 278 & 124 & 77 & 89 & & 4 & 2 & 76 & 24 & 8 & 230 & 856 & 114 & 970 & $\mathrm{D}, \mathrm{M}, \mathrm{S}$ \\
\hline Aleocharinae_sp. 2 & & 2 & & 1 & & & & & & & & & 3 & & 3 & $\mathrm{D}, \mathrm{M}, \mathrm{S}$ \\
\hline Aleocharinae sp. 3 & 2 & 1 & 2 & 1 & & & & & & & & & 6 & & 6 & $\mathrm{D}, \mathrm{M}, \mathrm{S}$ \\
\hline
\end{tabular}




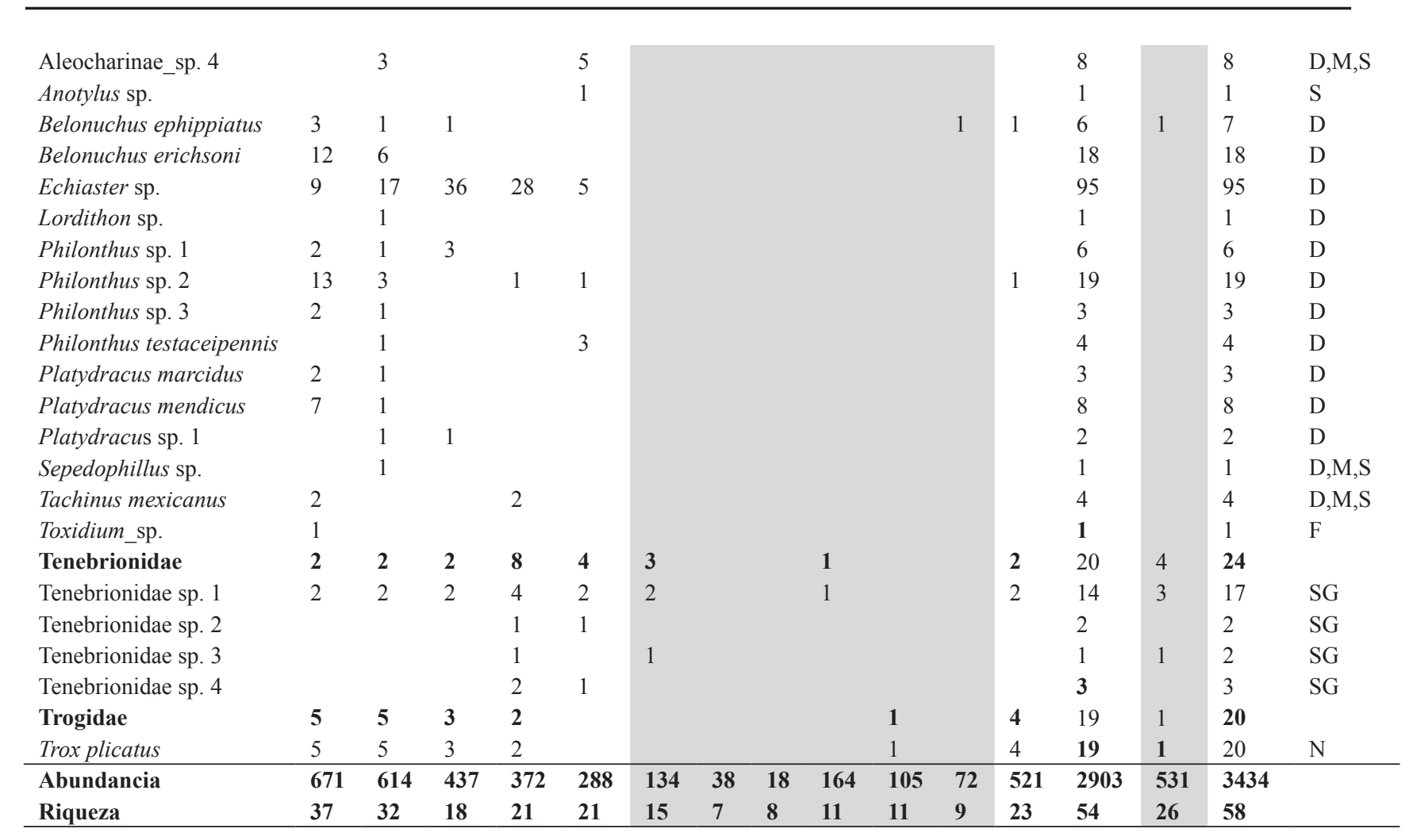

